\title{
Exciton Dynamics in Droplet Epitaxial Quantum Dots Grown on (311)A-Oriented Substrates
}

\author{
Marco Abbarchi ${ }^{1, *(\mathbb{D})}$, Takaaki Mano ${ }^{2}$, Takashi Kuroda ${ }^{2}\left(\mathbb{D}\right.$ and Kazuaki Sakoda ${ }^{2}(\mathbb{D}$ \\ 1 Aix Marseille University, Université de Toulon, CNRS, IM2NP Marseille, France \\ 2 Research Center for Functional Materials, National Institute for Materials Science, 1-1 Namiki, Tsukuba, \\ Ibaraki 305-0044, Japan; MANO.Takaaki@nims.go.jp (T.M.); KURODA.Takashi@nims.go.jp (T.K.); \\ SAKODA.Kazuaki@nims.go.jp (K.S.) \\ * Correspondence: marco.abbarchi@im2np.fr
}

Received: 31 July 2020; Accepted: 7 September 2020; Published: 14 September 2020

check for updates

\begin{abstract}
Droplet epitaxy allows the efficient fabrication of a plethora of 3D, III-V-based nanostructures on different crystalline orientations. Quantum dots grown on a (311)A-oriented surface are obtained with record surface density, with or without a wetting layer. These are appealing features for quantum dot lasing, thanks to the large density of quantum emitters and a truly $3 \mathrm{D}$ lateral confinement. However, the intimate photophysics of this class of nanostructures has not yet been investigated. Here, we address the main optical and electronic properties of s-shell excitons in individual quantum dots grown on (311)A substrates with photoluminescence spectroscopy experiments. We show the presence of neutral exciton and biexciton as well as positive and negative charged excitons. We investigate the origins of spectral broadening, identifying them in spectral diffusion at low temperature and phonon interaction at higher temperature, the presence of fine interactions between electron and hole spin, and a relevant heavy-hole/light-hole mixing. We interpret the level filling with a simple Poissonian model reproducing the power excitation dependence of the s-shell excitons. These results are relevant for the further improvement of this class of quantum emitters and their exploitation as single-photon sources for low-density samples as well as for efficient lasers for high-density samples.
\end{abstract}

Keywords: III-V quantum dots; droplet epitaxy; exciton dynamics; (311)A oriented substrate

\section{Introduction}

Low-dimensional nanostructures [1] have been widely employed for a plethora of applications ranging from sensing [2], light harvesting in solar cells [3], biology [4], and photonics [5-10]. Among the different categories, epitaxial quantum dots (QDs) stand as the best alternative for quantum devices thanks to their brightness, stability, and compatibility with photonic and electronic devices [7,8,10-12]. Within this class of QDs, droplet epitaxy (DE) $[10,13,14]$ and droplet etching $[9,15,16]$ (alternative growth protocols to Stranski-Krastanov for strain-free III-V-based semiconductor nanostructures), enabled the fabrication of state-of-the-art devices such as lasers [17-21] and quantum emitters, including single-photon sources [22-26] and entangled photons [9,27-30] with electrical injection [31]. The versatility of this method allowed to grow many different semiconductor alloys (GaInSb [32], AlGaAs [33-37], InGaAs [38-44], and InGaP [26,45,46]), forming a plethora of nanostructures [47] such as quantum dots (QDs); QDs diads [48-50]; multiple-concentric quantum rings [18,23,51-56]; coupled structures such as ring-on-a-disk [57], dot in-a-ring [58], or dot-on-a-disk [59]; as well as elongated structures such as nanowires [60]. This technique allows to independently tune the size and density of the nanostructures [61] and to grow them with or without a wetting layer [35,39,40,60,62-66], aspects that are not matched by the conventional Stranski-Krastanov approach based on strain [67]. 
Another aspect relevant for applications is the possibility to grow high-quality nanostructures on different substrate orientation, providing the ground for highly symmetric QDs on (111)A surfaces [26,27,35,36,41,42,46,61,68-71] (e.g., for entangled photon pairs generation) and for ultra-high density quantum wires [60] and QDs [19,32,38,63-65,72,73] formation on the highly anisotropic (311)A surface (e.g., for laser emission). This latter class of nanostructures grown on (311)A surface has not yet been thoroughly investigated and a clear assessment of the corresponding excitonic dynamics has not yet been reported.

In this paper, we show a detailed structural and optical characterization of individual QDs grown on the (311)A surface. We provide a clear-cut attribution of the main recombination lines observed in the photoluminescence (PL) spectrum to the s-shell excitons $[33,73,74]$ based on polarization-resolved PL measurements, power dependence, and line broadening measurements. Neutral exciton $X$ and biexciton $X X$, positive $X^{+}$and negative charged excitons $X^{-}$are characterized by the presence of fine interactions $[33,34,40,75]$ as well as by heavy-hole/light-hole mixing [76-80]. Their power dependence under laser excitation above barrier is well reproduced by a simple Poissonian model that precisely accounts for the main features and allows to estimate the excitonic capture volume [81]. Inhomogeneous line broadening at low temperature is ascribed to spectral diffusion [24,30,82-85] induced by the presence of charged defects nearby the QDs [86] and is specific to the excitonic complex in study $[87,88]$. Finally, at higher temperature, the photoluminescence of individual QDs broadens and quenches owing to phonon interactions [89-91].

\section{Materials and Methods}

\subsection{Sample Fabrication}

The sample was grown on a semi-insulating GaAs (311)A substrate by conventional solid-source molecular-beam epitaxy system (MBE32 by Riber). After the growth of a $2 \mu \mathrm{m}$ thick $\mathrm{Al}_{0.55} \mathrm{Ga}_{0.45} \mathrm{As}$ layer, a $136 \mathrm{~nm}$ thick $\mathrm{Al}_{0.26} \mathrm{Ga}_{0.74} \mathrm{As}$ core-layer was grown at $610{ }^{\circ} \mathrm{C}$. At the core layer center, $\mathrm{GaAs}$ QDs were formed by droplet epitaxy. On the $\mathrm{Al}_{0.26} \mathrm{Ga}_{0.74} \mathrm{As}$ surface, nominally 1.5 monolayers of $\mathrm{Ga}$ (at a growth speed of $\sim 0.1$ monolayers per second) was supplied without $\mathrm{As}_{4}$ flux at $275^{\circ} \mathrm{C}$ for the droplets formation. These droplets were then crystallized into GaAs QDs by supplying an As $\mathrm{Alux}_{4}$ $\left(2 \times 10^{-6}\right.$ Torr beam equivalent pressure) at $200^{\circ} \mathrm{C}$. The QDs were annealed at $400^{\circ} \mathrm{C}$ for 10 min under $\mathrm{As}_{4}$ flux supply without capping in order to improve the crystal quality. After annealing, the QDs were covered with a $30 \mathrm{~nm}$ thick $\mathrm{Al}_{0.26} \mathrm{Ga}_{0.74} \mathrm{As}$ capping layer at $400{ }^{\circ} \mathrm{C}$ and the rest of the $\mathrm{Al}_{0.26} \mathrm{Ga}_{0.74} \mathrm{As}$ $(38 \mathrm{~nm})$ layer was grown at $625^{\circ} \mathrm{C}$.

Once the entire growth sequence was completed, a rapid thermal annealing process was performed at $785{ }^{\circ} \mathrm{C}$ for $4 \mathrm{~min}$ in an $\mathrm{As}_{4}$ atmosphere to improve the optical quality. The effect of ex situ rapid thermal annealing step has been reported in [18,92]. From these studies it emerges that nanostructures crystallized into GaAs by a supply of low As flux show a dramatic increase of the corresponding emission intensity. Transmission electron microscopy [18] shows a negligible effect on the nanostructure morphology with a very small thickening of the wetting layer. In spite of the rather small interdiffusion coefficient between GaAs and AlAs [93], for high-temperature post-growth annealing, a modification of the PL band with a blue shift can be observed [92].

In order to characterize the QDs density and morphology, a similar sample was grown without capping, thus leaving the QDs exposed for otimic force microscopy investigation. For that purpose we used an atomic force microscope (AFM, SPA400 by Hitachi High-Tech) in non-contact mode.

\subsection{Optical Spectroscopy}

The photoluminescence (PL) of individual QDs was collected with a confocal-spectroscopic set-up (lateral resolution of $\sim 1 \mu \mathrm{m}) \mathrm{CW}$ laser excitation was performed above-barrier, at $532 \mathrm{~nm}(\sim 2.3 \mathrm{eV})$. The PL is then fed into a spectrometer and detected by a Si-based charge-coupled device (CCD) 
camera, allowing for a spectral resolution better than $50 \mu \mathrm{eV}$ in full width at half maximum (FWHM). All experiments were performed in a liquid helium cryostat between 10 and $100 \mathrm{~K}$.

\section{Results and Discussion}

We first provide a structural characterization of (311)A QDs via AFM study (Figure 1) in order to highlight their strongly anisotropic shape. Figure $1 \mathrm{a}$, b shows atomic force micrographs (AFM) of the sample after annealing at $400{ }^{\circ} \mathrm{C}$. Well-defined QDs are present with a density of approximately $5 \times 10^{9} / \mathrm{cm}^{2}$. The QD morphology is highly asymmetric, with a U-shape (Figure $1 \mathrm{~b}$ ). We attribute the formation mechanism of these asymmetric QDs to the low-intensity $\mathrm{As}_{4}$ supply for the crystallization of the droplets and surface asymmetry of the (311)A surface $[19,32,38,60,63-65,72]$. In this growth condition, the Ga droplets crystallization into GaAs is enhanced around the edge of the droplets. In the case of standard (100) surface, in which the oppositely oriented directions of [011] and [0-11] are equivalent, ring-like structures with central holes are formed [51,52]. On the (311)A surface, however, the oppositely oriented directions of [-233] and [2-3-3] are not equivalent, while the directions of [01-1] and [0-11] are equivalent. Thus, the crystallization is enhanced only in a particular direction ([-233]). As a result, U-shaped QDs are formed.
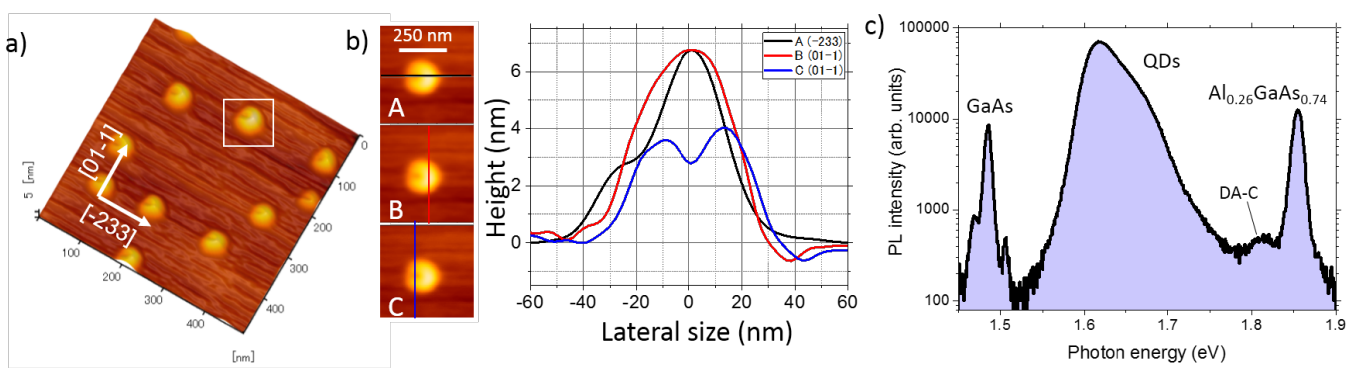

Figure 1. (a) Atomic force microscope micrograph representing a 3D view of uncapped GaAs quantam dots (QDs) grown on a (311)A oriented $\mathrm{Al}_{0.26} \mathrm{Ga}_{0.74} \mathrm{As}$ surface. The main crystallographic axes are highlighted. (b) Left panels: blow-up of a single QD from panel (a). A, B, and C highlight cuts in different crystallographic directions and points of the QD and are reported in the right panel. (c) Photoluminescence (PL) spectrum (in semi-log scale) at $5 \mathrm{~K}$ showing the emission of (respectively, from the low to the high energy side) the GaAs, the GaAs QDs, the carbon donor-acceptor (DA-C), and the $\mathrm{Al}_{0.26} \mathrm{Ga}_{0.74}$ As barrier layer.

Macro PL on the QDs ensemble taken at low temperature shows the different contributions from the GaAs substrate (at $\sim 1.48 \mathrm{eV}$ ), the $\mathrm{Al}_{0.26} \mathrm{Ga}_{0.74} \mathrm{As}$ barriers (at $\sim 1.85 \mathrm{eV}$ ) and the QDs in between, extending over a broad band from approximately $1.55 \mathrm{eV}$ up to $1.75 \mathrm{eV}$. Aside from this, we note a small PL peak at $\sim 1.82 \mathrm{eV}$. We attribute this emission to shallow centers due to carbon-related residual contamination (donor-acceptor carbon (DA-C)) that is commonly found in epitaxial QDs grown in molecular beam epitaxy reactors $[94,95]$.

\section{1. s-Shell Excitons}

Typical spectra of individual QDs on (311)A substrate appear structured in several sharp lines (Figure 2a). Although at the center of the macro-PL band (at approximately $1.62 \mathrm{eV}$ ) the spatial density of QDs is larger making the isolation of individual QDs more complicate, emission from single QDs in the full range from about $1.6 \mathrm{up}$ to $1.8 \mathrm{eV}$ is in principle possible [73]. In analogy to most III-V epitaxial QDs, including those fabricated via DE $[22,33,73,96]$, in the low excitation power regime, the s-shell excitons dominate the PL spectrum. We ascribe the brightest lines to the recombination of the neutral exciton X (one electron, $e$, and a heavy-hole, $h h$ ), neutral biexciton (XX, two $e$ and two $h h$ ), positive charged exciton (one $e$ and two $h h$ ), and to the negative charged exciton (two $e$ and a $h h$ ). This attribution is based on the study of the electron-hole fine interaction [33] (fine structure splitting), 
line broadening [82] (spectral diffusion), and level filling [81], as discussed in the following sections. Seldom have we also observed other sharp lines, such as M0 and M1 (Figure 2a), that likely involve carrier recombination from the p-shell. However, a precise attribution of these lines is not possible with this set of data and goes beyond the aim of this paper.
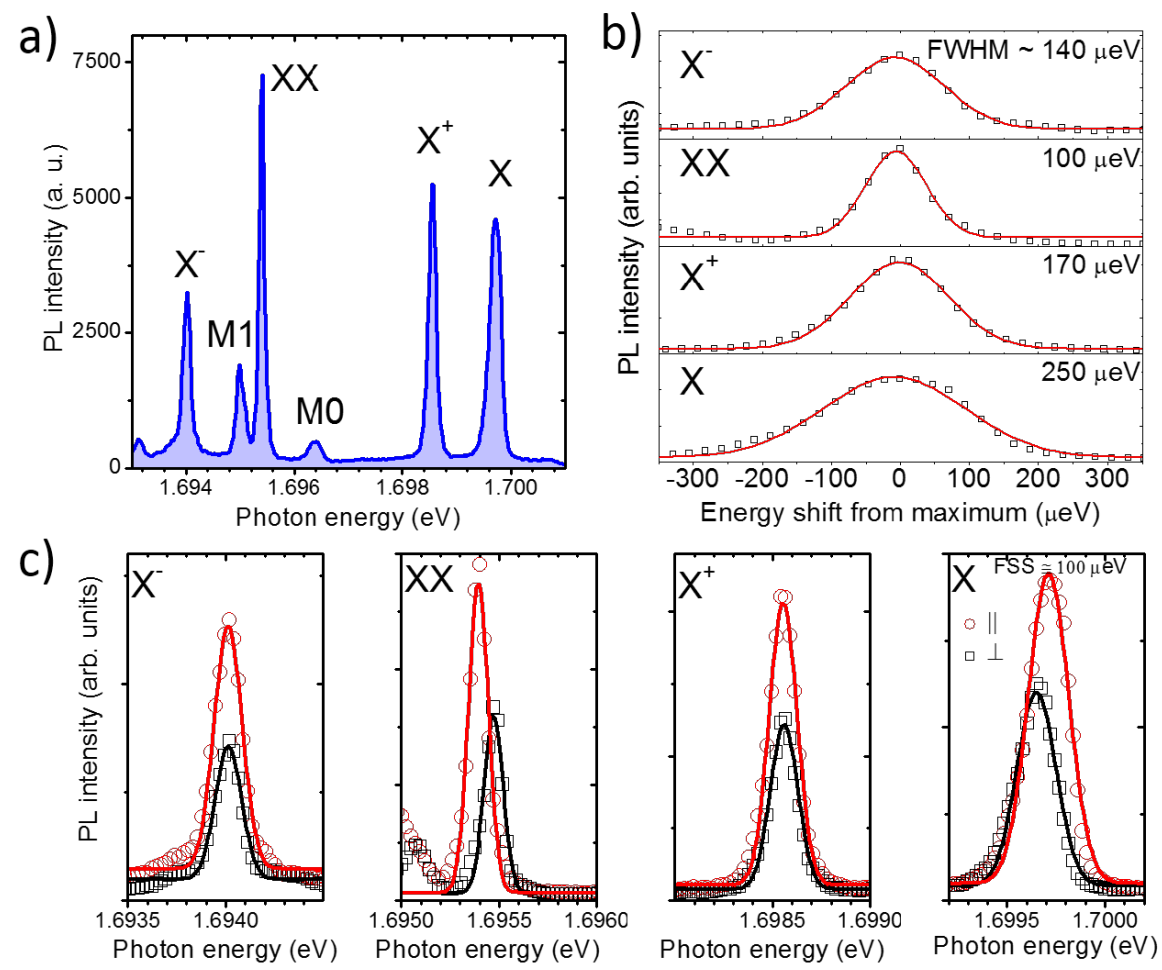

Figure 2. (a) PL spectrum of a single GaAs QDs sandwiched between (311) $\mathrm{A} \mathrm{Al}_{0.35} \mathrm{Ga}_{0.65} \mathrm{As}$ barrier layers. The labels on the most intense lines $\mathrm{X}, \mathrm{X}^{+}, \mathrm{XX}$, and $\mathrm{X}^{-}$highlight, respectively, the emission from the neutral exciton, the positive charged exciton, the neutral biexciton, and the negative charged exciton. M0 and M1 highlight other non-attributed multiexciton complexes. (b) Symbols: PL spectra of $\mathrm{X}, \mathrm{X}^{+}, \mathrm{XX}$, and $\mathrm{X}^{-}$(respectively, from the bottom to the top panel). Red lines are Gaussian fit to the data. The corresponding full width at half maximum (FWHM) is reported on each panel. (c) Linearly polarized components of $\mathrm{X}, \mathrm{X}^{+}, \mathrm{XX}$, and $\mathrm{X}^{-} \mathrm{PL}$ (respectively, from the right to the left panel). Red and black symbols indicate orthogonal polarization. The continuous lines are Gaussian fits. The fine structure splitting (FSS) measured from the $\mathrm{X}$ and XX components is $\sim 100 \mu \mathrm{eV}$.

\section{2. $e$-h Spin Fine Interaction: Fine Structure Splitting}

A first identification of the s-shell excitons is based on the study of the fine interaction between electron and $h$ h spin $[33,34,75,97]$ (the fine structure splitting (FSS), Figure 2a)). At intermediate laser excitation power (at approximately $420 \mathrm{nW}$ ) all the PL components in the spectrum are well visible (Figure 2a). At this power, by changing the polarization angle of the detected light, we monitor the emission energy of the PL. We observe for $X$ and XX a mirror-symmetric energy splitting (Figure 2c). $\mathrm{X}^{+}$and $\mathrm{X}^{-}$instead, do not feature any splitting.

This picture corresponds very well to what is commonly observed in strain-free, III-V QDs: the presence of geometrical asymmetries in the confining potential breaks the invariance of the Hamiltonian for rotations around the vertical growth axis $[33,34,37,75,78,97,98]$. More precisely, $e-h$ spin-spin interaction for the $\mathrm{X}$ state lifts the degeneracy of the corresponding energy level and leads to two linearly polarized PL components, depending on which recombination path is radiating. The XX state has overall zero spin for $e$ and $h h$ in the initial state and its FSS is completely determined by that of the $X$ state, towards which it relaxes. $X^{+}$and $X^{-}$can recombine with two possible energy equivalent paths and have no FSS. 
The measured FSS in this QD is of $100 \mu \mathrm{eV}$. In other QDs we measured values between 30 and $140 \mu \mathrm{eV}$ (not shown). These values are very large if compared with QDs grown on (111) substrates, where the three-fold symmetry of the crystal provides a more isotropic surface diffusion and a corresponding triangular or hexagonal nanostructure shape $[26,27,35,36,41,42,46,61,68-71]$. The origin of this splitting in (311)A QDs is attributed to asymmetries in the QDs shape that is affected by the anisotropy of the underlying crystal. The measured values of FSS are in line with those observed in (001) QDs in spite of the larger anisotropy of the (311)A surface [33,34,75,97]. Owing to the growth method in use, that allows for 3D growth of lattice-matched materials such as GaAs on AlGaAs, we exclude the presence of any strain in the structure that is a major origin of symmetry breaking (and thus FSS) in conventional Stranski-Krastanov III-V QDs [97].

\subsection{Polarization Intensity Anisotropy: Heavy-Hole Light-Hole Mixing}

We monitor the polarization anisotropy of $X$ and $X X$ split components observing the different intensity of the corresponding PL lines (Figure 2c)). A remarkable intensity change is visible for the two orthogonal polarized components (between $1 / 3$ and $1 / 2$, depending on the observed line, Figure 2c)) of $X$ and $X X$ as well as in the polarization intensity of $X^{+}$and $X^{-}$. We ascribe this difference to heavy-hole/light-hole (hh-lh) mixing [76-80,99]. This feature was first highlighted in DE QDs grown on (001)-oriented substrates and was explained as an effect of the different $h h$ and $l h$ bands dispersion in the different crystallographic directions [76]. While $e$ states can be approximately described as isotropic and parabolic bands, valence $h h$ and $t h$ bands are affected by strong anisotropy and different curvatures. In spite of the large energy splitting between $h h$ and $l h$ bands in $\mathrm{GaAs}$ (tens of meV), strain and corresponding piezoelectricity [77,79,80,99] (in SK QDs) or shape asymmetries [76,78] (in DE QDs) lead to a substantial mixing of the corresponding $h$ h and $t h$ states, providing different selection rules (uneven PL intensity) for the two recombination paths. Here, we observe differences in the PL intensity that are of the same order of those observed in the $(001)$ counterpart $[76,77,79,80,99]$.

\subsection{Line Broadening: Spectral Diffusion}

We address the origin of the line broadening by monitoring the line-shape and width of each s-shell excitonic species. By monitoring the line-shape and corresponding broadening of each PL component in the s-shell we observe, respectively, a common Gaussian envelope and a remarkably different full width at half maximum (FWHM; Figure 2b). The common origin of the line broadening in this class of QDs is spectral diffusion: in spite of the expected natural linewidth that should be of the order of a few $\mu \mathrm{eV}$ with a corresponding Lorentian lineshape $[82,84,85,96]$, the measured broadening values (tens to hundreds of $\mu \mathrm{eV}$ ) and Gaussian envelopes are the fingerprints of a fluctuating charged environment. Charging and un-charging of electron and holes traps nearby the QDs (within a few tens of $\mathrm{nm}$ ), produces a variable quantum confined Stark-shift of the excitonic energy levels [24,30,82-86]. A PL measurement lasting for a few seconds acquires many photons emitted at slightly different energy, thus providing a Gaussian lineshape. However, owing to the different $h h$ and $e$ confinement (larger penetration in the barrier for $e$ with respect to $h h$ ) and a screening of external electric field for many-particles states, the corresponding Stark-shift can be very different. It has been routinely reported a larger broadening of $X$ with respect to the other s-shell excitons, whereas the relative broadening of $\mathrm{XX}, \mathrm{X}^{+}$, and $\mathrm{X}^{-}$can be very different, depending on the carrier trapped and its position with respect to the QD $[24,82,95]$. As an example, the case in Figure $2 \mathrm{~b}$ seems to correspond to electrons confined in the plane of the QD [82]. However, this picture can be quite different from a QD to another, owing to the randomness and local changes of the extrinsic disorder.

State-of-the-art droplet epitaxial QDs have been shown capable of extremely narrow PL bands, in the few $\mu \mathrm{eV}$ range [96] and record fidelity in X-XX photon entanglement $[27,29]$ that requires a high degree of coherence. It is also worth mentioning that alternative methods, such as droplet etching, have been proven capable of high quality PL lines with a line broadening of about $20 \mu \mathrm{eV}$ [16] and entanglement [9]. In this case of (311)A QDs, in spite of the similar high-temperature process 
used to grow high quality droplet epitaxial nanostructures on (001) substrates, the line broadening is rather large, comparable to lower quality QDs grown at lower temperature [33,82,83,90]. A precise assessment of this phenomenology would require a more systematic study and a clear-cut attribution of the larger defectivity is not possible. We observe that these QDs have a very peculiar shape, with a gap on one side. This feature may lead to a more pronounced tunneling of the excitonic wavefunction in the barrier, where most of the defects are located. Exploring the nearby environment may lead to a more effective interaction with the charged defects that in turn lead to a larger broadening.

\subsection{Power Dependence: Level Filling}

Let us now address the level filling of the s-shell excitons by power dependent measurements (Figure 3). At low power, only two lines, $X$ and $X^{+}$, are visible with a dominant intensity of $X$, while when increasing the power also $X^{-}$and, soon after, $X X$ appears. At larger power, $X$ and $X^{+}$swap their relative intensity and then quench. $X X$ and $X^{-}$reach their maximum and quench at very large power. M0 and M1 follows a similar dynamics of $X^{-}$and $X X$, respectively. At lower energy, other peaks appear together with a broad background that then extends to all the spectrum. These latter features are interpreted as the effect of multi-excitons recombining from the p-shell and the coupling with the continuum of states above the confined energy levels. For the sake of thoroughness, we note a slight red-shift of the PL intensity at large excitation power. This effect is interpreted as a heating of the sample. It is also worth noting that the PL intensity of the s-shell excitons can be followed over more than 3 orders of magnitude of the excitation power, meaning that the capture and recombination processes are very efficient in this sample.
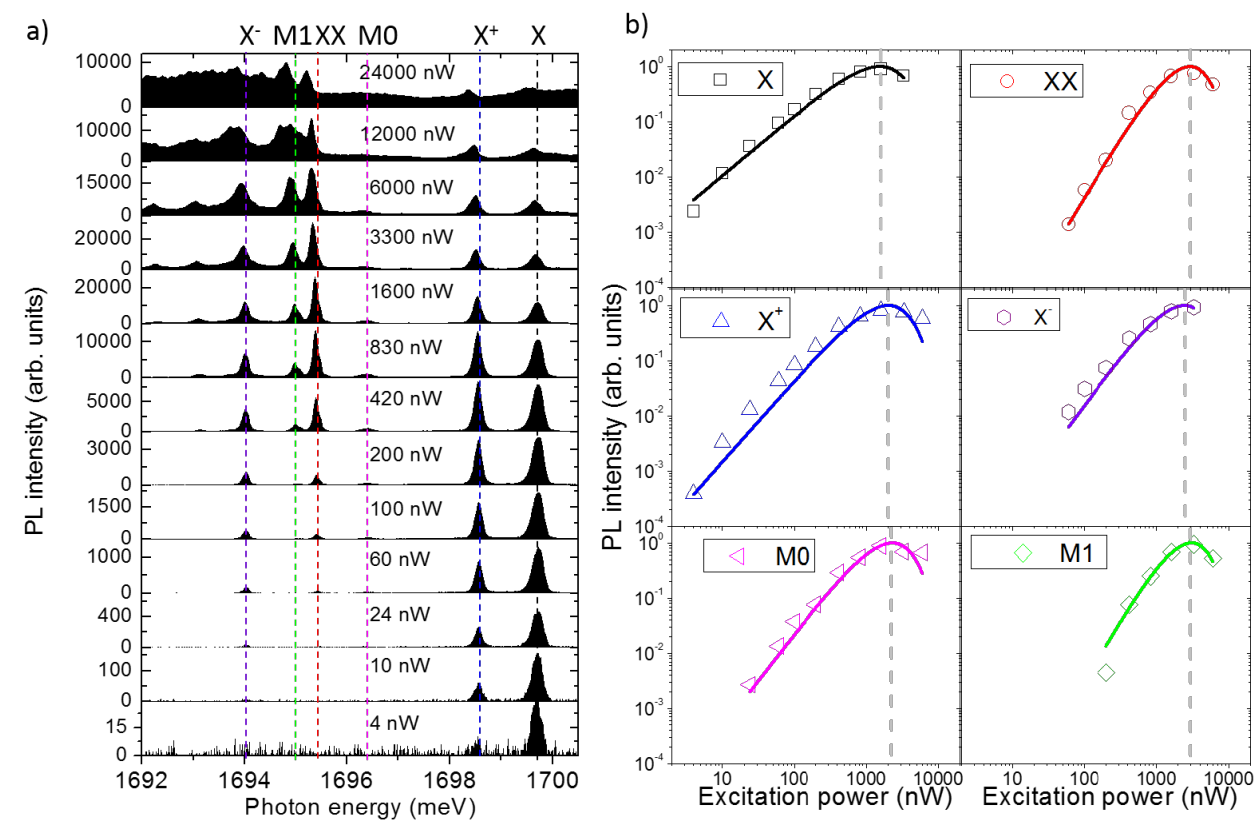

Figure 3. (a) PL spectra from low to high excitation power for the same QD shown in Figure 2. The incident power is highlighted on each panel. The vertical dashed lines act as guides for the eyes. (b) Evolution of the six main PL lines as a function of the incident excitation power. The PL intensity has been normalized to the maximum for each component. Each panel refers to a specific exciton complex recombination as highlighted by the corresponding labels. Symbols are the experimental data whereas lines are Poissonian fits.

A simple but meaningful description of the level filling of individual QDs can be provided by a model based on the assumption that carrier capture and recombination are random processes [100]. By assuming an infinite set of confined levels in the nanostructure, that obviously constitutes a rough approximation, the model can be further simplified to a Poissonian dependence of the 
PL intensity [81,100]. In spite of its simplicity, this modeling accounts extremely well for power dependence [81] and recombination dynamics [46,101] of the s-shell excitons. In this model, we assume that the PL intensity $I_{P L}$ of a given energy level is proportional to the occupation probability $N_{n}$ of that level:

$$
I_{P L} \propto N_{n}
$$

The Poissonian model for level filling predicts that the occupation of a certain level with $n$ excitons $N_{n}$ is provided by

$$
N_{n}=\frac{<n>^{n}}{n !} e^{-<n>}
$$

Thus, for $n=1, N_{1}$ describes the level filling of $\mathrm{X}$, for $n=2, N_{2}$ describes the level filling of $X X$, etc. $\langle n\rangle$ is the average number of exciton created in the QD. This number is obtained averaging over many capture/recombination cycles and under steady state excitation and is defined as $\langle n\rangle=N_{R} \tau_{r} / \tau_{c}$, where $N_{R}$ is the number of e-h pairs created in the reservoir, $\tau_{r}$ is the exciton recombination rate, and $\tau_{c}$ the exciton capture rate. Provided that $\tau_{r}$ and $\tau_{c}$ lie in the ps to ns range and the integration time for a PL measurement last about one second, this hypothesis is well satisfied.

A final phenomenological assumption for $\langle n\rangle$ that allows to link the experimental data to this Poissonian model is that

$$
<n>=\beta P_{e x c}^{\alpha}
$$

where $\alpha$ and $\beta$ are constants characterizing the capture mechanism. Note that in the vast majority of works on QDs, the level filling of the s-shell excitons is simply provided by Equation (3), with $\alpha(X) \sim 1$ for $X$, and $\alpha(X X) \sim 2$ for $X X$. However, this oversimplified assumption does not account for saturation and quenching of the PL lines under CW excitation [31,33,69,102].

The Poissonian model well reproduces the power dependence of $X$ and $X X$ that are extracted by integrating in energy their PL line up to $1600 \mathrm{nW}$ after background subtraction (Figure 3b). Fitting the data using Equations (2) and (3), we obtain $\alpha_{X}=1.1$ (very close to 1 as expected) and a simultaneous fit of $X$ and $X X$ with the same parameters. The saturation power of $X$ is $\sim 1500 \mathrm{nW}$ and about two times larger for $X X$.

Although the simple Poissonian model allows only for neutral excitons capture $(n=1,2, \ldots)$ and in principle cannot account for charged complexes, a nice fitting of the data can be recovered by assuming half-integer numbers [81]. In this case, the model is modified by adding a phenomenological coefficient $\gamma$ to $\alpha_{X}$ :

$$
N_{n}=\frac{\left(<n>^{n}\right)^{\gamma}}{n !} e^{-<n>}
$$

Thus, for instance, $\gamma=1$ corresponds to a filling dynamics similar to that of the neutral exciton $X$ state, whereas $\gamma=1.5$ is one and an half exciton (between $X$ and $X X$ ), that is, a charged exciton (with an additional spectator charge induced by optical pumping). By using Equation 4 we can nicely fit the power dependence of $X^{+}$and $X^{-}$by keeping unchanged the parameters $\alpha$ and $\beta$ that were used to fit $X$ and $X X$ (Figure 3b). We obtain $\alpha\left(X^{+}\right)=1.3 \alpha(X)$ and $\alpha\left(X^{-}\right)=1.6 \alpha(X)$ that are very close to 1.5.

Note that, in this specific QD, the power dependence of $X^{+}$and $X^{+}$is superlinear with respect to $X$. In addition to this, their corresponding saturation power is larger with respect to $X$. This is not always the case, and a very similar power dependence of charged and neutral complexes can be observed $(\gamma \sim 1)$ [81]. These differences can be ascribed to the nature of the spectator charge in the QD at the moment of $e-h$ recombination: the presence of the extra carrier can be ascribed either to residual doping of the heterostructure or to optical injection. In the first case, when the extra carrier comes from a donor/acceptor impurity, the corresponding power dependence of the trions follows a trend similar to that one of the neutral counterpart $(\gamma \sim 1$ and same saturation power of $X)$ [81]. If instead 
the spectator carrier is optically injected in the QD, a super-linear trend and a larger saturation power with respect to $X$ is expected. In this latter case, a quasi-Poissonian law with $\gamma>1$ well reproduces the experimental data.

We note that also the dynamics of M0 and M1 with incident power can be nicely accounted for by this pseudo-Poissonian model providing $\gamma(M 0)=1.5$ and $\gamma(M 1)=2.1$. These values of $\gamma$ that are larger than 1 account for the super-linear dependence on the incident power and support the multi-exciton nature of these energy levels. Based on the similarities of these lines with those reported in droplet epitaxial QDs grown on (111)A substrates (without a wetting layer as in this case), and on the superlinear power dependence, we speculate that M0 and M1 could be related to recombination of doubly charged excitons [36] or recombination in presence of a carrier in the p-shell [103]. A more conclusive assessment of these lines would require charge tunable devices and magneto-photoluminescence. However, these characterizations go well beyond the aim of this work.

To conclude this section, we estimate the QD capture volume for excitons [81]. This can be deduced from the saturation power of the X level: at that power (of approximately $1500 \mathrm{nW}$ distributed over a diameter of $1 \mu \mathrm{m}$ ) the number of carriers created in the host matrix around the QD provides a steady occupation of the $X$ level by one exciton. Thus, the inverse of the carrier density injected in the semiconductor at that saturation power represents the capture volume. Assuming a penetration depth of the laser light of $\sim 1 \mu \mathrm{m}$, an excitonic recombination lifetime of about $1 \mathrm{~ns}$ and considering the QD as a sphere, we can roughly deduce a capture radius of the order of $50 \mathrm{~nm}$. This value is rather large with respect to the physical size of the QD, that is of the order of a few $\mathrm{nm}$. We also observe that this value is perfectly in line with those measured in the (001) DE QDs counterpart [81] as well as in other kind of quantum emitters in III-V [95] and in group IV materials [104].

\subsection{Temperature Dependence}

We now address the PL properties of individual QDs as a function of temperature (Figure 4). Bright PL emission can be observed well above liquid nitrogen temperature (Figure 4). A quadratic red-shift of the exciton emission is observed, as expected for this class of nanostructures that follows the empirical Varshini law (Figure 4a). At the same time, a strong quenching of the PL intensity of about two orders of magnitude is observed when passing from 10 to $90 \mathrm{~K}$.
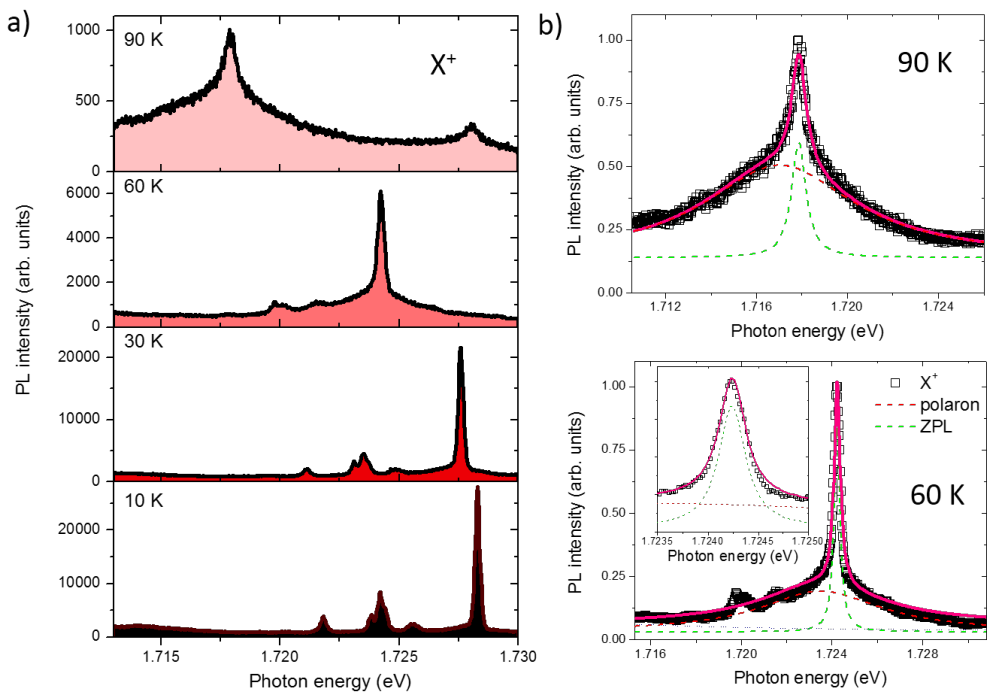

Figure 4. (a) Selected PL spectra from 10 to $90 \mathrm{~K}$ of a single QD with a bright $\mathrm{X}^{+}$emission. (b) Bottom panel: detail of the spectrum from $\mathrm{A}$ at $60 \mathrm{~K}$ with Lorentzian fits of the zero phonon line and the polaron band. The inset shows a blow up of the zero phonon line (ZPL). Top panel: detail of the spectrum at $90 \mathrm{~K}$ with Lorentzian fits. The measurements have been performed exciting with a laser power of $7000 \mathrm{nW}$. 
The low-temperature spectrum (up to about $30 \mathrm{~K}$ ), as discussed in the previous sections, shows sharp lines attributed to the s-shell excitons and is well described by a Gaussian envelope (Figure 2) [82]. At higher temperature instead, when the phonon population plays an important role, we observe the onset of a broad Lorentzian-shaped pedestal below the original sharp line that can be now well approximated with a Lorentzian envelope (Figure $4 \mathrm{~b}$ ) $[89,90,105]$. The relative intensity of this broad pedestal increases and dominates over the sharp central line. This phenomenology corresponds well to what has been thoroughly explained by using the Huang-Rhys formalism for (001) DE QDs as well as for SK QDs $[89,90,105]$ : the broad band is interpreted as a superposition of acoustic phonon replicas (polaron) whereas the sharp central line emission is assigned to the zero phonon line (ZPL).

\section{Conclusions}

In conclusion, we showed that droplet epitaxial QDs grown on the highly anisotropic (311)A surface are characterized by bright PL lines emitting at visible frequency. These lines correspond well to the picture of s-shell excitons recombination in III-V nanostructures. We measured a relatively large fine structure splitting $(10-100 \mu \mathrm{eV})$ of the neutral exciton $X$, that is justified by the anisotropic shape of these QDs. Inhomogeneous line broadening lies at $100 \mu \mathrm{eV}$ and is ascribed to spectral diffusion originating from the fluctuating charged environment. This broadening is specific of each excitonic species and reflects the nature and position of the trapped carriers. Level filling of the s-shell excitons is well accounted for by a simple Poissonian model that allows to describe the power dependence of the main PL lines and to estimate an excitonic capture volume much larger than the physical size of the QD. Finally, a bright PL emission is still visible well above liquid nitrogen temperature.

Overall, these results account for the relevance of this class of QDs, where $X, X X$, and $X^{+}$emissions can be followed for approximately 3 orders of magnitude of excitation power below saturation and up to relatively large temperature that are appealing features for applications as single-photon sources. The low-temperature linewidth is rather large with respect to state-of-the-art droplet epitaxial quantum dots, and in principle it could be improved by using alternative fabrication processes at higher temperature. Exploiting resonant $[85,106,107]$ or two-photon [29] laser excitation the issues of broadening and spin fine structures can be overcome as shown in similar systems. Another possible way to overcome the large fine structure splitting observed in these QDs for quantum entanglement with $X$ and $X X$ recombination is exploiting the time reordering scheme $[95,108,109]$. In this case, a negligibly small $X X$ binding energy is required. Although this condition has not yet been reported in (311)A QDs [73], exploiting the lack of a wetting layer (and thus a large lateral confinement) and growing smaller QDs could lead to this special configuration of the energy levels. Finally, spectral selection of entangled photon can be achieved embedding the emitter in a cavity [110] or applying external strain to compensate for the asymmetric quantum dot shape [111].

Author Contributions: T.M. fabricated the samples and performed the structural characterization. T.K. conceived and supervised the spectroscopic measurements, M.A. performed the spectroscopic measurements and redacted the paper, K.S. supervised and coordinated the research. All authors contributed to the redaction of the paper, have read and agreed to the published version of the manuscript.

Conflicts of Interest: The authors declare no conflicts of interest.

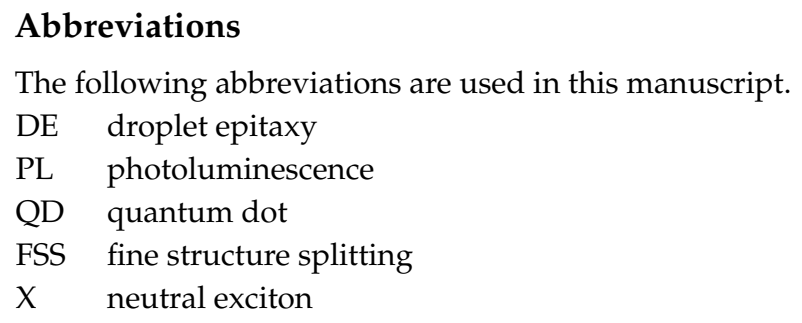


$\mathrm{X}^{+} \quad$ positive charged exciton

XX neutral biexciton

$X^{-} \quad$ negative charged exciton

FWHM full width at half maximum

\section{References}

1. Alizadeh-Ghodsi, M.; Pourhassan-Moghaddam, M.; Zavari-Nematabad, A.; Walker, B.; Annabi, N.; Akbarzadeh, A. State-of-the-Art and Trends in Synthesis, Properties, and Application of Quantum Dots-Based Nanomaterials. Part. Part. Syst. Charact. 2019, 36, 1800302. [CrossRef]

2. Ciotta, E.; Paoloni, S.; Richetta, M.; Prosposito, P.; Tagliatesta, P.; Lorecchio, C.; Venditti, I.; Fratoddi, I.; Casciardi, S.; Pizzoferrato, R. Sensitivity to heavy-metal ions of unfolded fullerene quantum dots. Sensors 2017, 17, 2614. [CrossRef]

3. Bomm, J.; Büchtemann, A.; Chatten, A.J.; Bose, R.; Farrell, D.J.; Chan, N.L.; Xiao, Y.; Slooff, L.H.; Meyer, T.; Meyer, A.; et al. Fabrication and full characterization of state-of-the-art quantum dot luminescent solar concentrators. Sol. Energy Mater. Sol. Cells 2011, 95, 2087-2094. [CrossRef]

4. Rodzik-Czałka, Ł.; Lewandowska-Łańcucka, J.; Gatta, V.; Venditti, I.; Fratoddi, I.; Szuwarzyński, M.; Romek, M.; Nowakowska, M. Nucleobases functionalized quantum dots and gold nanoparticles bioconjugates as a fluorescence resonance energy transfer (FRET) system-Synthesis, characterization and potential applications. J. Colloid Interface Sci. 2018, 514, 479-490. [CrossRef]

5. Demir, H.V.; Nizamoglu, S.; Erdem, T.; Mutlugun, E.; Gaponik, N.; Eychmüller, A. Quantum dot integrated LEDs using photonic and excitonic color conversion. Nano Today 2011, 6, 632-647. [CrossRef]

6. Michler, P. Single Semiconductor Quantum Dots; Springer: Berlin/Heidelberg, Germany, 2009; Volume 231.

7. Strauf, S.; Hennessy, K.; Rakher, M.; Choi, Y.S.; Badolato, A.; Andreani, L.; Hu, E.; Petroff, P.; Bouwmeester, D. Self-tuned quantum dot gain in photonic crystal lasers. Phys. Rev. Lett. 2006, 96, 127404. [CrossRef]

8. Lodahl, P. Quantum-dot based photonic quantum networks. Quantum Sci. Technol. 2017, 3, 013001. [CrossRef]

9. Huber, D.; Reindl, M.; Huo, Y.; Huang, H.; Wildmann, J.S.; Schmidt, O.G.; Rastelli, A.; Trotta, R. Highly indistinguishable and strongly entangled photons from symmetric GaAs quantum dots. Nat. Commun. 2017, 8, 1-7. [CrossRef]

10. Gurioli, M.; Wang, Z.; Rastelli, A.; Kuroda, T.; Sanguinetti, S. Droplet epitaxy of semiconductor nanostructures for quantum photonic devices. Nat. Mater. 2019, 18, 799-810. [CrossRef]

11. Lodahl, P.; Stobbe, S. Solid-state quantum optics with quantum dots in photonic nanostructures. Nanophotonics 2013, 2, 39-55. [CrossRef]

12. Beveratos, A.; Abram, I.; Gérard, J.M.; Robert-Philip, I. Quantum optics with quantum dots. Eur. Phys. J. 2014, 68, 377. [CrossRef]

13. Mantovani, V.; Sanguinetti, S.; Guzzi, M.; Grilli, E.; Gurioli, M.; Watanabe, K.; Koguchi, N. Low density GaAs/ AlGaAs quantum dots grown by modified droplet epitaxy. J. Appl. Phys. 2004, 96, 4416-4420. [CrossRef]

14. Wu, J.; Wang, Z.M. Droplet epitaxy for advanced optoelectronic materials and devices. J. Phys. Appl. Phys. 2014, 47, 173001. [CrossRef]

15. Atkinson, P.; Zallo, E.; Schmidt, O. Independent wavelength and density control of uniform GaAs/AlGaAs quantum dots grown by infilling self-assembled nanoholes. J. Appl. Phys. 2012, 112, 054303. [CrossRef]

16. Huo, Y.; Rastelli, A.; Schmidt, O. Ultra-small excitonic fine structure splitting in highly symmetric quantum dots on GaAs (001) substrate. Appl. Phys. Lett. 2013, 102, 152105. [CrossRef]

17. Mano, T.; Kuroda, T.; Yamagiwa, M.; Kido, G.; Sakoda, K.; Koguchi, N. Lasing in Ga As/ Al Ga As self-assembled quantum dots. Appl. Phys. Lett. 2006, 89, 183102. [CrossRef]

18. Mano, T.; Kuroda, T.; Mitsuishi, K.; Yamagiwa, M.; Guo, X.J.; Furuya, K.; Sakoda, K.; Koguchi, N. Ring-shaped GaAs quantum dot laser grown by droplet epitaxy: Effects of post-growth annealing on structural and optical properties. J. Cryst. Growth 2007, 301, 740-743. [CrossRef]

19. Mano, T.; Kuroda, T.; Mitsuishi, K.; Nakayama, Y.; Noda, T.; Sakoda, K. Ga As / Al Ga As quantum dot laser fabricated on GaAs (311) A substrate by droplet epitaxy. Appl. Phys. Lett. 2008, 93, 203110. [CrossRef] 
20. Jo, M.; Mano, T.; Sakoda, K. Lasing in ultra-narrow emission from GaAs quantum dots coupled with a two-dimensional layer. Nanotechnology 2011, 22, 335201. [CrossRef]

21. Jo, M.; Mano, T.; Sakoda, K. Electrical Lasing in GaAs Quantum Dots Grown by Droplet Epitaxy. Adv. Opt. Mater. Opt. Soc. Am. 2012, ITh5B-6. [CrossRef]

22. Kuroda, T.; Abbarchi, M.; Mano, T.; Watanabe, K.; Yamagiwa, M.; Kuroda, K.; Sakoda, K.; Kido, G.; Koguchi, N.; Mastrandrea, C.; et al. Photon correlation in GaAs self-assembled quantum dots. Appl. Phys. Express 2008, 1, 042001. [CrossRef]

23. Abbarchi, M.; Mastrandrea, C.; Vinattieri, A.; Sanguinetti, S.; Mano, T.; Kuroda, T.; Koguchi, N.; Sakoda, K.; Gurioli, M. Photon antibunching in double quantum ring structures. Phys. Rev. B 2009, 79, 085308. [CrossRef]

24. Abbarchi, M.; Kuroda, T.; Mano, T.; Gurioli, M.; Sakoda, K. Bunched photon statistics of the spectrally diffusive photoluminescence of single self-assembled GaAs quantum dots. Phys. Rev. B 2012, 86, 115330. [CrossRef]

25. Benyoucef, M.; Zuerbig, V.; Reithmaier, J.P.; Kroh, T.; Schell, A.W.; Aichele, T.; Benson, O. Single-photon emission from single InGaAs/GaAs quantum dots grown by droplet epitaxy at high substrate temperature. Nanoscale Res. Lett. 2012, 7, 1-5. [CrossRef]

26. Kumano, H.; Harada, T.; Suemune, I.; Nakajima, H.; Kuroda, T.; Mano, T.; Sakoda, K.; Odashima, S.; Sasakura, H. Stable and efficient collection of single-photons emitted from a semiconductor quantum dot into a single-mode optical fiber. Appl. Phys. Express 2016, 9, 032801. [CrossRef]

27. Kuroda, T.; Mano, T.; Ha, N.; Nakajima, H.; Kumano, H.; Urbaszek, B.; Jo, M.; Abbarchi, M.; Sakuma, Y.; Sakoda, K.; et al. Symmetric quantum dots as efficient sources of highly entangled photons: Violation of Bell's inequality without spectral and temporal filtering. Phys. Rev. B 2013, 88, 041306. [CrossRef]

28. Kumano, H.; Nakajima, H.; Kuroda, T.; Mano, T.; Sakoda, K.; Suemune, I. Nonlocal biphoton generation in a Werner state from a single semiconductor quantum dot. Phys. Rev. B 2015, 91, 205437. [CrossRef]

29. Basso Basset, F.; Bietti, S.; Reindl, M.; Esposito, L.; Fedorov, A.; Huber, D.; Rastelli, A.; Bonera, E.; Trotta, R.; Sanguinetti, S. High-yield fabrication of entangled photon emitters for hybrid quantum networking using high-temperature droplet epitaxy. Nano Lett. 2018, 18, 505-512. [CrossRef]

30. Ramírez, H.Y.; Chou, Y.L.; Cheng, S.J. Effects of electrostatic environment on the electrically triggered production of entangled photon pairs from droplet epitaxial quantum dots. Sci. Rep. 2019, 9, 1-10. [CrossRef]

31. Ha, N.; Mano, T.; Kuroda, T.; Sakuma, Y.; Sakoda, K. Current-injection quantum-entangled-pair emitter using droplet epitaxial quantum dots on GaAs (111) A. Appl. Phys. Lett. 2019, 115, 083106. [CrossRef]

32. Kawazu, T.; Noda, T.; Mano, T.; Jo, M.; Sakaki, H. Effects of antimony flux on morphology and photoluminescence spectra of GaSb quantum dots formed on GaAs by droplet epitaxy. J. Nonlinear Opt. Phys. Mater. 2010, 19, 819-826. [CrossRef]

33. Abbarchi, M.; Mastrandrea, C.; Kuroda, T.; Mano, T.; Sakoda, K.; Koguchi, N.; Sanguinetti, S.; Vinattieri, A.; Gurioli, M. Exciton fine structure in strain-free $\mathrm{GaAs} / \mathrm{Al}_{0.3} \mathrm{Ga}_{0.7} \mathrm{As}$ quantum dots: Extrinsic effects. Phys. Rev. B 2008, 78, 125321. [CrossRef]

34. Plumhof, J.; Křápek, V.; Wang, L.; Schliwa, A.; Bimberg, D.; Rastelli, A.; Schmidt, O. Experimental investigation and modeling of the fine structure splitting of neutral excitons in strain-free $\mathrm{GaAs}_{\mathrm{A}} / \mathrm{Al}_{\mathrm{x}} \mathrm{Ga}_{1-\mathrm{x}}$ As quantum dots. Phys. Rev. B 2010, 81, 121309. [CrossRef]

35. Mano, T.; Abbarchi, M.; Kuroda, T.; McSkimming, B.; Ohtake, A.; Mitsuishi, K.; Sakoda, K. Self-assembly of symmetric GaAs quantum dots on (111) A substrates: Suppression of fine-structure splitting. Appl. Phys. Express 2010, 3, 065203. [CrossRef]

36. Bouet, L.; Vidal, M.; Mano, T.; Ha, N.; Kuroda, T.; Durnev, M.; Glazov, M.; Ivchenko, E.; Marie, X.; Amand, T.; et al. Charge tuning in [111] grown GaAs droplet quantum dots. Appl. Phys. Lett. 2014, 105, 082111. [CrossRef]

37. Yeo, I.; Kim, D.; Lee, K.T.; Kim, J.S.; Song, J.D.; Park, C.H.; Han, I.K. Comparative Chemico-Physical Analyses of Strain-Free GaAs $/ \mathrm{Al}_{0.3} \mathrm{Ga}_{0.7}$ As Quantum Dots Grown by Droplet Epitaxy. Nanomaterials 2020, 10, 1301. [CrossRef]

38. Saidi, F.; Bouzaiene, L.; Sfaxi, L.; Maaref, H. Growth conditions effects on optical properties of InAs quantum dots grown by molecular beam epitaxy on GaAs (113) A substrate. J. Lumin. 2012, 132, 289-292. [CrossRef] 
39. Zuerbig, V.; Bugaew, N.; Reithmaier, J.P.; Kozub, M.; Musiał, A.; Sęk, G.; Misiewicz, J. Growth-Temperature Dependence of Wetting Layer Formation in High Density InGaAs/GaAs Quantum Dot Structures Grown by Droplet Epitaxy. Jpn. J. Appl. Phys. 2012, 51, 085501. [CrossRef]

40. Skiba-Szymanska, J.; Stevenson, R.M.; Varnava, C.; Felle, M.; Huwer, J.; Müller, T.; Bennett, A.J.; Lee, J.P.; Farrer, I.; Krysa, A.B.; et al. Universal growth scheme for quantum dots with low fine-structure splitting at various emission wavelengths. Phys. Rev. Appl. 2017, 8, 014013. [CrossRef]

41. Mano, T.; Mitsuishi, K.; Ha, N.; Ohtake, A.; Castellano, A.; Sanguinetti, S.; Noda, T.; Sakuma, Y.; Kuroda, T.; Sakoda, K. Growth of metamorphic InGaAs on GaAs (111) a: Counteracting lattice mismatch by inserting a thin InAs interlayer. Cryst. Growth Des. 2016, 16, 5412-5417. [CrossRef]

42. Ha, N.; Mano, T.; Wu, Y.N.; Ou, Y.W.; Cheng, S.J.; Sakuma, Y.; Sakoda, K.; Kuroda, T. Wavelength extension beyond $1.5 \mu \mathrm{m}$ in symmetric InAs quantum dots grown on InP (111) A using droplet epitaxy. Appl. Phys. Exp. 2016, 9, 101201. [CrossRef]

43. Fuster, D.; Abderrafi, K.; Alén, B.; González, Y.; Wewior, L.; González, L. InAs nanostructures grown by droplet epitaxy directly on InP (001) substrates. J. Cryst. Growth 2016, 434, 81-87. [CrossRef]

44. Sala, E.M.; Na, Y.I.; Godsland, M.; Trapalis, A.; Heffernan, J. InAs/InP Quantum Dots in Etched Pits by Droplet Epitaxy in Metalorganic Vapor Phase Epitaxy. Phys. Status Solidi (Rrl) Rapid Res. Lett. 2020, 14, 2000173. [CrossRef]

45. Prongjit, P.; Ratanathammaphan, S.; Ha, N.; Mano, T.; Sakoda, K.; Kuroda, T. Type-II recombination dynamics of tensile-strained GaP quantum dots in GaAs grown by droplet epitaxy. Appl. Phys. Lett. 2016, 109, 171902. [CrossRef]

46. Ha, N.; Mano, T.; Dubos, S.; Kuroda, T.; Sakuma, Y.; Sakoda, K. Single photon emission from droplet epitaxial quantum dots in the standard telecom window around a wavelength of $1.55 \mu \mathrm{m}$. Appl. Phys. Exp. 2020, 13, 025002. [CrossRef]

47. Bietti, S.; Bocquel, J.; Adorno, S.; Mano, T.; Keizer, J.G.; Koenraad, P.M.; Sanguinetti, S. Precise shape engineering of epitaxial quantum dots by growth kinetics. Phys. Rev. B 2015, 92, 075425. [CrossRef]

48. Yamagiwa, M.; Mano, T.; Kuroda, T.; Tateno, T.; Sakoda, K.; Kido, G.; Koguchi, N.; Minami, F. Self-assembly of laterally aligned GaAs quantum dot pairs. Appl. Phys. Lett. 2006, 89, 113115. [CrossRef]

49. Wang, Z.M.; Holmes, K.; Mazur, Y.I.; Ramsey, K.A.; Salamo, G.J. Self-organization of quantum dot pairs by high-temperature droplet epitaxy. Nanoscale Res. Lett. 2006, 1, 57. [CrossRef]

50. Sablon, K.; Lee, J.; Wang, Z.M.; Shultz, J.; Salamo, G. Configuration control of quantum dot molecules by droplet epitaxy. Appl. Phys. Lett. 2008, 92, 203106. [CrossRef]

51. Mano, T.; Koguchi, N. Nanometer-scale GaAs ring structure grown by droplet epitaxy. J. Cryst. Growth 2005, 278, 108-112. [CrossRef]

52. Mano, T.; Kuroda, T.; Sanguinetti, S.; Ochiai, T.; Tateno, T.; Kim, J.; Noda, T.; Kawabe, M.; Sakoda, K.; Kido, G.; et al. Self-assembly of concentric quantum double rings. Nano Lett. 2005, 5, 425-428. [CrossRef]

53. Somaschini, C.; Bietti, S.; Koguchi, N.; Sanguinetti, S. Fabrication of multiple concentric nanoring structures. Nano Lett. 2009, 9, 3419-3424. [CrossRef] [PubMed]

54. Shwartz, N.L.; Vasilenko, M.A.; Nastovjak, A.G.; Neizvestny, I.G. Concentric GaAs nanorings formation by droplet epitaxy-Monte Carlo simulation. Comput. Mater. Sci. 2018, 141, 91-100. [CrossRef]

55. Sanguinetti, S.; Mano, T.; Kuroda, T. Self-assembled semiconductor quantum ring complexes by droplet epitaxy: Growth and physical properties. In Physics of Quantum Rings; Springer: Berlin/Heidelberg, Germany, 2018; pp. 187-228.

56. Heyn, C.; Zocher, M.; Hansen, W. Functionalization of Droplet Etching for Quantum Rings. In Physics of Quantum Rings; Springer: Berlin/Heidelberg, Germany, 2018; pp. 139-162.

57. Somaschini, C.; Bietti, S.; Sanguinetti, S.; Koguchi, N.; Fedorov, A. Self-assembled GaAs/AlGaAs coupled quantum ring-disk structures by droplet epitaxy. Nanotechnology 2010, 21, 125601. [CrossRef] [PubMed]

58. Somaschini, C.; Bietti, S.; Koguchi, N.; Sanguinetti, S. Coupled quantum dot-ring structures by droplet epitaxy. Nanotechnology 2011, 22, 185602. [CrossRef] [PubMed]

59. Elborg, M.; Noda, T.; Mano, T.; Kuroda, T.; Yao, Y.; Sakuma, Y.; Sakoda, K. Self-assembly of vertically aligned quantum ring-dot structure by Multiple Droplet Epitaxy. J. Cryst. Growth 2017, 477, 239-242. [CrossRef]

60. Jo, M.; Keizer, J.G.; Mano, T.; Koenraad, P.M.; Sakoda, K. Self-assembly of GaAs quantum wires grown on (311) A substrates by droplet epitaxy. Appl. Phys. Exp. 2011, 4, 055501. [CrossRef] 
61. Ohtake, A.; Ha, N.; Mano, T. Extremely high-and low-density of Ga droplets on GaAs $\{111\}$ A, B: Surface-polarity dependence. Cryst. Growth Des. 2015, 15, 485-488. [CrossRef]

62. Sanguinetti, S.; Watanabe, K.; Tateno, T.; Wakaki, M.; Koguchi, N.; Kuroda, T.; Minami, F.; Gurioli, M. Role of the wetting layer in the carrier relaxation in quantum dots. Appl. Phys. Lett. 2002, 81, 613-615. [CrossRef]

63. Mano, T.; Noda, T.; Kuroda, T.; Sanguinetti, S.; Sakoda, K. Self-assembled GaAs quantum dots coupled with GaAs wetting layer grown on GaAs (311) A by droplet epitaxy. Phys. Status Solidi C 2011, 8, 257-259. [CrossRef]

64. Keizer, J.; Jo, M.; Mano, T.; Noda, T.; Sakoda, K.; Koenraad, P. Structural atomic-scale analysis of $\mathrm{GaAs} / \mathrm{AlGaAs}$ quantum wires and quantum dots grown by droplet epitaxy on a (311) A substrate. Appl. Phys. Lett. 2011, 98, 193112. [CrossRef]

65. Keizer, J.; Koenraad, P. Atomic-scale analysis of self-assembled quantum dots by cross-sectionalscanning, tunneling microscopy, and atom probe tomography. In Quantum Dots: Optics, Electron Transport and Future Applications; Cambridge University Press: Cambridge, UK, 2012; pp. 41-60.

66. Shahzadeh, M.; Sabaeian, M. Wetting layer-assisted modification of in-plane-polarized transitions in strain-free GaAs/AlGaAs quantum dots. Superlattices Microstruct. 2014, 75, 514-522. [CrossRef]

67. Sautter, K.E.; Vallejo, K.D.; Simmonds, P.J. Strain-driven quantum dot self-assembly by molecular beam epitaxy. J. Appl. Phys. 2020, 128, 031101. [CrossRef]

68. Jo, M.; Mano, T.; Abbarchi, M.; Kuroda, T.; Sakuma, Y.; Sakoda, K. Self-limiting growth of hexagonal and triangular quantum dots on (111) A. Cryst. Growth Des. 2012, 12, 1411-1415. [CrossRef]

69. Liu, X.; Ha, N.; Nakajima, H.; Mano, T.; Kuroda, T.; Urbaszek, B.; Kumano, H.; Suemune, I.; Sakuma, Y.; Sakoda, K. Vanishing fine-structure splittings in telecommunication-wavelength quantum dots grown on (111) A surfaces by droplet epitaxy. Phys. Rev. B 2014, 90, 081301. [CrossRef]

70. Trapp, A.; Reuter, D. Formation of self-assembled GaAs quantum dots via droplet epitaxy on misoriented GaAs (111) B substrates. J. Vac. Sci. Technol. Nanotechnol. Microelectron. Mater. Process. Meas. Phenom. 2018, 36, 02D106. [CrossRef]

71. Bietti, S.; Basset, F.B.; Tuktamyshev, A.; Bonera, E.; Fedorov, A.; Sanguinetti, S. High-temperature droplet epitaxy of symmetric GaAs/AlGaAs quantum dots. Sci. Rep. 2020, 10, 1-10. [CrossRef]

72. Mano, T.; Kuroda, T.; Mitsuishi, K.; Noda, T.; Sakoda, K. High-density GaAs/AlGaAs quantum dots formed on GaAs (311) A substrates by droplet epitaxy. J. Cryst. Growth 2009, 311, 1828-1831. [CrossRef]

73. Abbarchi, M.; Kuroda, T.; Mano, T.; Sakoda, K.; Mastrandrea, C.A.; Vinattieri, A.; Gurioli, M.; Tsuchiya, T. Energy renormalization of exciton complexes in GaAs quantum dots. Phys. Rev. B 2010, 82, 201301. [CrossRef]

74. Accanto, N.; Minari, S.; Cavigli, L.; Bietti, S.; Isella, G.; Vinattieri, A.; Sanguinetti, S.; Gurioli, M. Kinetics of multiexciton complex in gaas quantum dots on si. Appl. Phys. Lett. 2013, 102, 053109. [CrossRef]

75. Tong, H.; Wu, M. Theory of excitons in cubic III-V semiconductor GaAs, InAs and GaN quantum dots: Fine structure and spin relaxation. Phys. Rev. B 2011, 83, 235323. [CrossRef]

76. Belhadj, T.; Amand, T.; Kunold, A.; Simon, C.M.; Kuroda, T.; Abbarchi, M.; Mano, T.; Sakoda, K.; Kunz, S.; Marie, X.; et al. Impact of heavy hole-light hole coupling on optical selection rules in GaAs quantum dots. Appl. Phys. Lett. 2010, 97, 051111. [CrossRef]

77. Lin, C.H.; You, W.T.; Chou, H.Y.; Cheng, S.J.; Lin, S.D.; Chang, W.H. Anticorrelation between the splitting and polarization of the exciton fine structure in single self-assembled InAs/GaAs quantum dots. Phys. Rev. B 2011, 83, 075317. [CrossRef]

78. Liao, Y.H.; Liao, C.C.; Ku, C.H.; Chang, Y.C.; Cheng, S.J.; Jo, M.; Kuroda, T.; Mano, T.; Abbarchi, M.; Sakoda, K. Geometrical impact on the optical polarization of droplet epitaxial quantum dots. Phys. Rev. B 2012, 86, 115323. [CrossRef]

79. Plumhof, J.; Trotta, R.; Křápek, V.; Zallo, E.; Atkinson, P.; Kumar, S.; Rastelli, A.; Schmidt, O.G. Tuning of the valence band mixing of excitons confined in GaAs/AlGaAs quantum dots via piezoelectric-induced anisotropic strain. Phys. Rev. B 2013, 87, 075311. [CrossRef]

80. Luo, J.W.; Bester, G.; Zunger, A. Supercoupling between heavy-hole and light-hole states in nanostructures. Phys. Rev. B 2015, 92, 165301. [CrossRef]

81. Abbarchi, M.; Mastrandrea, C.; Kuroda, T.; Mano, T.; Vinattieri, A.; Sakoda, K.; Gurioli, M. Poissonian statistics of excitonic complexes in quantum dots. J. Appl. Phys. 2009, 106, 053504. [CrossRef] 
82. Abbarchi, M.; Troiani, F.; Mastrandrea, C.; Goldoni, G.; Kuroda, T.; Mano, T.; Sakoda, K.; Koguchi, N.; Sanguinetti, S.; Vinattieri, A.; et al. Spectral diffusion and line broadening in single self-assembled GaAs / AlGaAs quantum dot photoluminescence. Appl. Phys. Lett. 2008, 93, 162101. [CrossRef]

83. Mano, T.; Abbarchi, M.; Kuroda, T.; Mastrandrea, C.; Vinattieri, A.; Sanguinetti, S.; Sakoda, K.; Gurioli, M. Ultra-narrow emission from single GaAs self-assembled quantum dots grown by droplet epitaxy. Nanotechnology 2009, 20, 395601. [CrossRef]

84. Kuroda, K.; Kuroda, T.; Watanabe, K.; Mano, T.; Kido, G.; Koguchi, N.; Sakoda, K. Distribution of exciton emission linewidth observed for GaAs quantum dots grown by droplet epitaxy. J. Lumin. 2010, 130, 2390-2393. [CrossRef]

85. Nguyen, H.S.; Sallen, G.; Abbarchi, M.; Ferreira, R.; Voisin, C.; Roussignol, P.; Cassabois, G.; Diederichs, C. Photoneutralization and slow capture of carriers in quantum dots probed by resonant excitation spectroscopy. Phys. Rev. B 2013, 87, 115305. [CrossRef]

86. Ha, N.; Mano, T.; Chou, Y.L.; Wu, Y.N.; Cheng, S.J.; Bocquel, J.; Koenraad, P.M.; Ohtake, A.; Sakuma, Y.; Sakoda, K.; et al. Size-dependent line broadening in the emission spectra of single GaAs quantum dots: Impact of surface charge on spectral diffusion. Phys. Rev. B 2015, 92, 075306. [CrossRef]

87. Schimpf, C.; Reindl, M.; Klenovskỳ, P.; Fromherz, T.; Da Silva, S.F.C.; Hofer, J.; Schneider, C.; Höfling, S.; Trotta, R.; Rastelli, A. Resolving the temporal evolution of line broadening in single quantum emitters. Optics Express 2019, 27, 35290-35307. [CrossRef] [PubMed]

88. Reigue, A.; Hostein, R.; Voliotis, V. Resonance fluorescence of a single semiconductor quantum dot: The impact of a fluctuating electrostatic environment. Semicond. Sci. Technol. 2019, 34, 113001. [CrossRef]

89. Sanguinetti, S.; Mano, T.; Oshima, M.; Tateno, T.; Wakaki, M.; Koguchi, N. Temperature dependence of the photoluminescence of InGaAs/GaAs quantum dot structures without wetting layer. Appl. Phys. Lett. 2002, 81, 3067-3069. [CrossRef]

90. Abbarchi, M.; Gurioli, M.; Vinattieri, A.; Sanguinetti, S.; Bonfanti, M.; Mano, T.; Watanabe, K.; Kuroda, T.; Koguchi, N. Phonon sideband recombination kinetics in single quantum dots. J. Appl. Phys. 2008, 104, 023504. [CrossRef]

91. Lee, S.e.; Yeo, I.; Jo, M.K.; Jeong, Y.W.; Kim, T.G.; Kim, J.S.; Yi, K.S.; Han, I.K.; Song, J.D. Exciton-phonon coupling channels in a 'strain-free'GaAs droplet epitaxy single quantum dot. Curr. Appl. Phys. 2018, 18, 829-833. [CrossRef]

92. Sanguinetti, S.; Mano, T.; Gerosa, A.; Somaschini, C.; Bietti, S.; Koguchi, N.; Grilli, E.; Guzzi, M.; Gurioli, M.; Abbarchi, M. Rapid thermal annealing effects on self-assembled quantum dot and quantum ring structures. J. Appl. Phys. 2008, 104, 113519. [CrossRef]

93. Schlesinger, T.; Kuech, T. Determination of the interdiffusion of Al and Ga in undoped (Al, Ga) As/GaAs quantum wells. Appl. Phys. Lett. 1986, 49, 519-521. [CrossRef]

94. Pavesi, L.; Guzzi, M. Photoluminescence of $\mathrm{Al}_{\mathrm{x}} \mathrm{Ga}_{1-\mathrm{x}}$ As alloys. J. Appl. Phys. 1994, 75, 4779-4842. [CrossRef]

95. Dotti, N.; Sarti, F.; Bietti, S.; Azarov, A.; Kuznetsov, A.; Biccari, F.; Vinattieri, A.; Sanguinetti, S.; Abbarchi, M.; Gurioli, M. Germanium-based quantum emitters towards a time-reordering entanglement scheme with degenerate exciton and biexciton states. Phys. Rev. B 2015, 91, 205316. [CrossRef]

96. Abbarchi, M.; Kuroda, T.; Duval, R.; Mano, T.; Sakoda, K. Scanning Fabry-Pérot interferometer with largely tuneable free spectral range for high resolution spectroscopy of single quantum dots. Rev. Sci. Instruments 2011, 82, 073103. [CrossRef] [PubMed]

97. Trotta, R.; Zallo, E.; Ortix, C.; Atkinson, P.; Plumhof, J.; Van den Brink, J.; Rastelli, A.; Schmidt, O. Universal recovery of the energy-level degeneracy of bright excitons in InGaAs quantum dots without a structure symmetry. Phys. Rev. Lett. 2012, 109, 147401. [CrossRef] [PubMed]

98. Adorno, S.; Bietti, S.; Sanguinetti, S. Annealing induced anisotropy in GaAs/AlGaAs quantum dots grown by droplet epitaxy. J. Cryst. Growth 2013, 378, 515-518. [CrossRef]

99. Fras, F.; Bernardot, F.; Eble, B.; Bernard, M.; Siarry, B.; Miard, A.; Lemaitre, A.; Testelin, C.; Chamarro, M. The role of heavy-light-hole mixing on the optical initialization of hole spin in InAs quantum dots. J. Phys. Condens. Matter 2013, 25, 202202. [CrossRef]

100. Grundmann, M.; Bimberg, D. Theory of random population for quantum dots. Phys. Rev. B 1997, 55, 9740. [CrossRef] 
101. Kuroda, T.; Belhadj, T.; Abbarchi, M.; Mastrandrea, C.; Gurioli, M.; Mano, T.; Ikeda, N.; Sugimoto, Y.; Asakawa, K.; Koguchi, N.; et al. Bunching visibility for correlated photons from single GaAs quantum dots. Phys. Rev. B 2009, 79, 035330. [CrossRef]

102. Tighineanu, P.; Daveau, R.; Lee, E.H.; Song, J.D.; Stobbe, S.; Lodahl, P. Decay dynamics and exciton localization in large GaAs quantum dots grown by droplet epitaxy. Phys. Rev. B 2013, 88, 155320. [CrossRef]

103. Durnev, M.; Vidal, M.; Bouet, L.; Amand, T.; Glazov, M.; Ivchenko, E.; Zhou, P.; Wang, G.; Mano, T.; Ha, N.; et al. Magnetospectroscopy of excited states in charge-tunable GaAs/AlGaAs [111] quantum dots. Phys. Rev. B 2016, 93, 245412. [CrossRef]

104. Beaufils, C.; Redjem, W.; Rousseau, E.; Jacques, V.; Kuznetsov, A.Y.; Raynaud, C.; Voisin, C.; Benali, A.; Herzig, T.; Pezzagna, S.; et al. Optical properties of an ensemble of G-centers in silicon. Phys. Rev. B 2018, 97, 035303. [CrossRef]

105. Favero, I.; Cassabois, G.; Ferreira, R.; Darson, D.; Voisin, C.; Tignon, J.; Delalande, C.; Bastard, G.; Roussignol, P.; Gérard, J. Acoustic phonon sidebands in the emission line of single InAs/GaAs quantum dots. Phys. Rev. B 2003, 68, 233301. [CrossRef]

106. Nguyen, H.S.; Sallen, G.; Voisin, C.; Roussignol, P.; Diederichs, C.; Cassabois, G. Optically gated resonant emission of single quantum dots. Phys. Rev. Lett. 2012, 108, 057401. [CrossRef]

107. Proux, R.; Maragkou, M.; Baudin, E.; Voisin, C.; Roussignol, P.; Diederichs, C. Measuring the photon coalescence time window in the continuous-wave regime for resonantly driven semiconductor quantum dots. Phys. Rev. Lett. 2015, 114, 067401. [CrossRef]

108. Troiani, F.; Tejedor, C. Entangled photon pairs from a quantum dot cascade decay: The effect of time reordering. Phys. Rev. B 2008, 78, 155305. [CrossRef]

109. Avron, J.; Bisker, G.; Gershoni, D.; Lindner, N.; Meirom, E.; Warburton, R. Entanglement on demand through time reordering. Phys. Rev. Lett. 2008, 100, 120501. [CrossRef] [PubMed]

110. Benson, O.; Santori, C.; Pelton, M.; Yamamoto, Y. Regulated and entangled photons from a single quantum dot. Phys. Rev. Lett. 2000, 84, 2513. [CrossRef]

111. Huber, D.; Reindl, M.; Da Silva, S.F.C.; Schimpf, C.; Martín-Sánchez, J.; Huang, H.; Piredda, G.; Edlinger, J.; Rastelli, A.; Trotta, R. Strain-tunable GaAs quantum dot: A nearly dephasing-free source of entangled photon pairs on demand. Phys. Rev. Lett. 2018, 121, 033902. [CrossRef]

(C) 2020 by the authors. Licensee MDPI, Basel, Switzerland. This article is an open access article distributed under the terms and conditions of the Creative Commons Attribution (CC BY) license (http:/ / creativecommons.org/licenses/by/4.0/). 